\title{
Oxidación de trans-2-hexen-1-ol empleando materiales mesoporosos
}

\section{Oxidation of trans-2-hexen-1-ol using mesoporous materials}

Presentación: 06/10/2020

\section{Doctorando:}

\section{Belén Melisa Viola}

Centro de Investigación y Tecnología Química, Facultad Regional Córdoba, Universidad Tecnológica Nacional - Argentina bviola@frc.utn.edu.ar

\section{Director/a:}

\section{Sandra Graciela Casuscelli}

Co-director/a:

\section{Analía Laura Cánepa \\ Griselda Alejandra Eimer}

\section{Resumen}

En este trabajo se estudió la reacción de oxidación del alcohol trans-2-hexen-1-ol utilizando catalizadores del tipo MCM-41. Para ello, se sintetizaron sólidos mesoporosos modificados con titanio que fueron caracterizados por área específica, DRX, UV-Vis RD, FTIR e ICP. Los sólidos mostraron buen ordenamiento estructural y elevada área específica. En el Ti-MCM-41 no se observaron óxidos metálicos, lo que sugiere una buena dispersión del Ti en la estructura. Los materiales sintetizados fueron evaluados catalíticamente en la reacción de oxidación de trans-2-hexen-1-ol en fase líquida, a $70{ }^{\circ} \mathrm{C}$, utilizando $\mathrm{H}_{2} \mathrm{O}_{2}$ como oxidante y acetonitrilo como solvente. Los productos de reacción fueron identificados mediante CG-Masa y cuantificados empleando factores de respuesta. Los resultados mostraron que la incorporación de Ti en la matriz mesoporosa incrementó la conversión del sustrato de 1.69\% mol a 4.29\% mol. Luego, se evaluó el efecto producido al variar la relación molar sustrato/oxidante empleando Ti-MCM-41. Con una relación igual a 1, se logró una conversión máxima de $8.26 \% \mathrm{~mol}$, con un rendimiento de $7.64 \%$ mol y una selectividad de $85.22 \%$ mol al 2-hexenal.

Palabras clave: oxidación, trans-2-hexen-1-ol, materiales mesoporosos

\begin{abstract}
In this work, the oxidation reaction of alcohol trans-2-hexen-1-ol was studied using catalysts of the MCM-41 type. Mesoporous solids were synthesized, pure and modified with titanium, which were characterized by specific area, XRD, UV-Vis RD, FTIR and ICP. The solids showed good structural ordering and high specific area. Metal oxides were not observed in Ti-MCM-41, suggesting a good dispersion of $\mathrm{Ti}$ in the structure. The synthesized materials were catalytically evaluated in the oxidation reaction of trans-2-hexen-1-ol in the liquid phase, at $70{ }^{\circ} \mathrm{C}, \mathrm{using} \mathrm{H}_{2} \mathrm{O}_{2}$ as oxidant and acetonitrile as solvent. The reaction products were identified by GC-Mass and quantified using the response factor. The results showed that the incorporation of $\mathrm{Ti}$ in the mesoporous matrix increased the conversion of the substrate from $1.69 \% \mathrm{~mol}$ to $4.29 \% \mathrm{~mol}$. Then, the effect produced by varying the substrate/oxidant molar ratio using Ti-MCM-41 was evaluated. A maximum conversion of $8.26 \% \mathrm{~mol}$ with a selectivity and yield to 2-hexenal of $85.22 \% \mathrm{~mol}$ and $7.64 \% \mathrm{~mol}$ respectively was achieved employed a molar ratio equal to 1.
\end{abstract}

Keywords: oxidation, trans-2-hexen-1-ol, mesoporous materials 


\section{Introducción}

El trans-2-hexen-1-ol es un compuesto orgánico volátil que se encuentra en la naturaleza. Pertenece al grupo de C6 que se generan en todos los tejidos verdes de las plantas ante situaciones de daño o estrés (Farag y Paré, 2002; Pang, 2015). También se encuentra presente en frutos como el kiwi (Talens et al., 2003), los arándanos (Du et al., 2014) y damascos (Gokbulut y Karabulut, 2012), entre otros. La reacción de oxidación del trans-2-hexen-1-ol presenta importancia desde el punto de vista industrial, ya que los productos obtenidos por la oxidación del grupo alcohol, epoxidación de la olefina u oxidaciones sucesivas de los mismos, dan lugar a la formación de intermediarios de interés agroquímico, farmacéutico y para la industria química en general (Sienel et al., 2008).

Los materiales mesoporosos del tipo MCM-41 presentan una estructura hexagonal con un arreglo regular de poros. Entre sus principales características se encuentran su elevada área específica y el tamaño de sus poros, que permiten la difusión de moléculas orgánicas voluminosas. La incorporación de metales de transición en estos tamices moleculares tiene interés desde el punto de vista catalítico. De hecho, el desarrollo de materiales con ordenamiento de mesoporos que contengan diferentes átomos en las paredes del poro ha abierto nuevas posibilidades para el uso de los tamices moleculares en el campo de la catálisis (Farzaneh et al., 2004). Así, el Ti-MCM-41 ha sido utilizado en reacciones de oxidación de alquenos, alcoholes y cetonas, además de emplearse en reacciones de epoxidación utilizando peróxido de hidrógeno como oxidante (Wróblewska et al., 2009). La ventaja de emplear $\mathrm{H}_{2} \mathrm{O}_{2}$ no sólo se debe a su bajo costo y a su elevado porcentaje de oxígeno activo sino también a que genera agua y oxígeno como subproductos de reacción, considerándose un oxidante eco-compatible.

En este trabajo se presentan los resultados obtenidos en la reacción de oxidación del alcohol trans-2-hexen-1-ol en fase líquida, utilizando peróxido de hidrógeno como oxidante y catalizadores del tipo MCM-41.

\section{Experimental}

\section{Síntesis de catalizadores}

La síntesis de los catalizadores se realizó aplicando el método sol-gel. Se utilizó tetraetil ortosilicato (TEOS) como fuente de silicio, bromuro de cetiltrimetilamonio como agente director de estructura (CTABr) y $\mathrm{NaOH}$ para ajustar el pH. Las relaciones molares empleadas fueron: $\mathrm{Si} /$ metal $=60,0 \mathrm{H} / \mathrm{Si}=0.5, \mathrm{CTABr} / \mathrm{Si}=0.12$ y $\mathrm{H}_{2} \mathrm{O} / \mathrm{Si}=132$. La solución preparada se mantuvo en agitación $4 \mathrm{~h}$ a temperatura ambiente y $3 \mathrm{~h}$ más a $70{ }^{\circ} \mathrm{C}$ para obtener la matriz silícea pura. Para sintetizar el material con titanio, a la solución preparada se le incorporó butóxido de titanio $\mathrm{Ti}\left(\mathrm{OC}_{4} \mathrm{H}_{9}\right)_{4}$ como fuente del metal, y se mantuvo $7 \mathrm{~h}$ en agitación a temperatura ambiente. Los sólidos obtenidos fueron filtrados, lavados con agua destilada hasta $\mathrm{pH}$ neutro y colocados en una estufa a $60^{\circ} \mathrm{C}$ durante $12 \mathrm{~h}$. Para eliminar el surfactante, los materiales se calcinaron en flujo de $\mathrm{N}_{2}$ hasta alcanzar $500{ }^{\circ} \mathrm{C}$ durante $6 \mathrm{~h}$ (rampa de $2{ }^{\circ} \mathrm{C} / \mathrm{min}$ - flujo $45 \mathrm{~mL} / \mathrm{min}$ ) y luego bajo flujo de aire $(45 \mathrm{~mL} / \mathrm{min})$ a $500{ }^{\circ} \mathrm{C}$. Así se obtuvieron los denominados MCM-41 y Ti-MCM- 41 .

\section{Caracterización fisicoquímica}

Los sólidos obtenidos fueron caracterizados por área específica utilizando un Chemisorb de Micromeritics 2700. Para conocer la estructura de los materiales se estudiaron los patrones de difracción de rayos X (DRX) obtenidos en un difractómetro X'Pert Pro PANalytical con una configuración de $2 \theta$ entre $2^{\circ}$ y $70^{\circ}$. El análisis de UV-Visible con reflectancia difusa (UV-Vis RD) se realizó para comprender el entorno de coordinación de las especies metálicas. Para ello, se utilizó un espectrómetro Jasco V650 equipado con esfera integradora tipo RSA-PE-20, en el rango de 200-900

$\mathrm{nm}$. La espectroscopía de emisión óptica por plasma acoplado inductivamente (ICP-OES) se realizó para comprobar el contenido metálico en los materiales. Se empleó un espectrofotómetro Agilent Technologies 5110. 


\section{Evaluación catalítica}

Las reacciones se llevaron a cabo en un reactor batch de vidrio de $10 \mathrm{~mL}$ de capacidad conectado a un condensador de reflujo. La temperatura de reacción se mantuvo a $70{ }^{\circ} \mathrm{C}$ utilizando un baño termostatizado y la reacción se realizó bajo agitación magnética vigorosa durante $5 \mathrm{~h}$. Se empleó trans-2-hexen-1-ol como sustrato (Sigma Aldrich 96\%), peróxido de hidrógeno como oxidante (30\% p/p, Cicarelli) y acetonitrilo como solvente (Sintorgan 99,5\%). La relación molar de solvente/sustrato utilizada fue de 10 y la concentración de catalizador de $9 \mathrm{~g} / \mathrm{L}$. Ambos parámetros se mantuvieron constantes mientras que la relación sustrato/oxidante se varió entre 1/1,2/1 y 4/1. Se extrajeron muestras de reacción a distintos tiempos, considerando como tiempo cero a la muestra extraída previa a la adición del catalizador en el medio de reacción.

\section{Identificación y cuantificación}

Las muestras de reacción fueron filtradas con un filtro milipore y analizadas mediante cromatografía gaseosa empleando un cromatógrafo AGILENT 7820 A equipado con una columna HP-1 (30 m de longitud y 0,53 mm de diámetro) y un detector FID. Además, se utilizó un cromatógrafo gaseoso con doble detector de masas (TRIPLE CUADRUPOLO) GC-MS/MS Thermo Scientific modelo TSQ 8000 EVO con inyector automático. El porcentaje de cada componente en las muestras se calculó utilizando el método de normalización de área empleando factores de respuesta (Skoog et al., 2008). La conversión del alcohol, las selectividades y rendimientos para cada producto se calcularon aplicando las ecuaciones 1,2 y 3 respectivamente:

$$
\begin{aligned}
& \text { Conversión }(\%)=\frac{\text { mmoles de productos oxidados }}{\text { mmoles iniciales de reactivo }} * 100 \\
& \text { Selectividad }_{i}(\%)=\frac{\text { mmol de }_{\text {producto }}}{\text { mmol de productos totales }} * 100 \\
& \text { Rendimiento }_{i}(\%)=\text { Conversión }_{(\%)} * \text { Selectividad }_{i}
\end{aligned}
$$

\section{Resultados}

Caracterización fisicoquímica

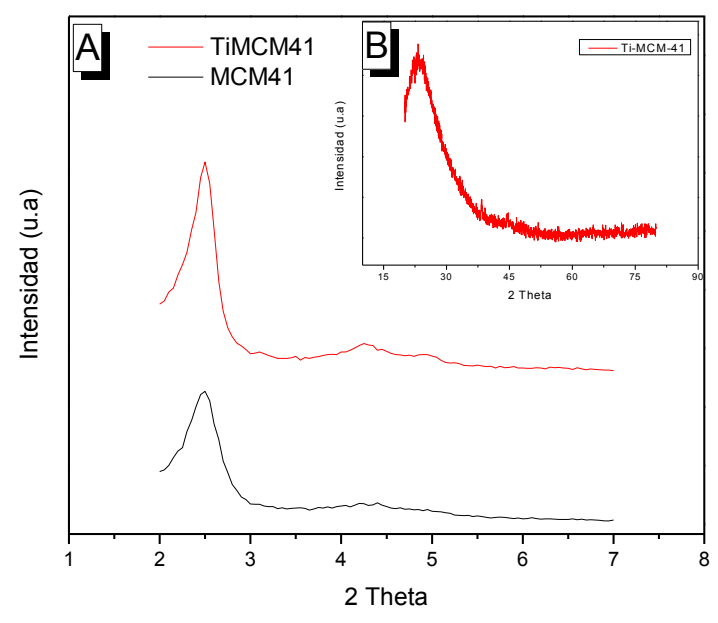

Figura 1: Patrones de DRX a bajo ángulo $(A)$ y a alto ángulo (B)
El valor de área medido en la matriz silícea pura fue de $1250 \mathrm{~m}^{2} / \mathrm{g}$ mientras que en el Ti-MCM-41 fue de $866 \mathrm{~m}^{2} / \mathrm{g}$. La disminución en el área probablemente se deba a un bloqueo parcial de los mesoporos generado por nanoclusters de Ti y/o partículas de óxidos de tamaño inferior a 5nm (Chanquía et al., 2016). Sin embargo, ambos materiales presentaron áreas elevadas, característica distintiva de la familia MCM-41.

En la Figura 1A se presentan los DRX a bajo ángulo, que indican un buen ordenamiento estructural en los sólidos ya que se observan los picos característicos de los planos (100), (110) y (200) presentes en las MCM-41 (Yang et al., 2013). En la Figura 1B se muestra el DRX a alto ángulo del Ti-MCM-41 donde se observa un hombro ubicado a $2 \theta=23.3^{\circ}$ representativo del carácter amorfo de estos materiales (Jia et al., 2019). No se observaron reflexiones pertenecientes a óxidos metálicos, lo que sugiere que el $\mathrm{Ti}$ se encuentra disperso en el material, y en caso de existir dichas especies, serían clusters y/o partículas de óxido de tamaño inferior al límite de detección de DRX (5 nm). 


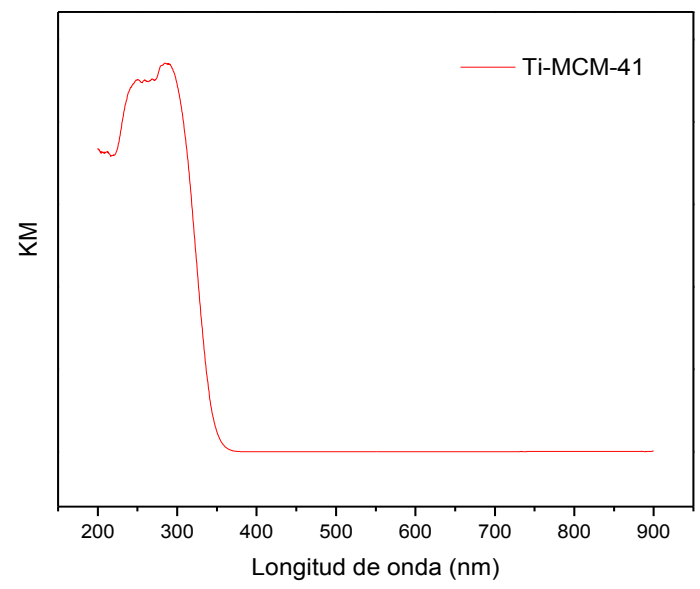

Figura 2: Espectro UV-Vis de Ti-MCM-41

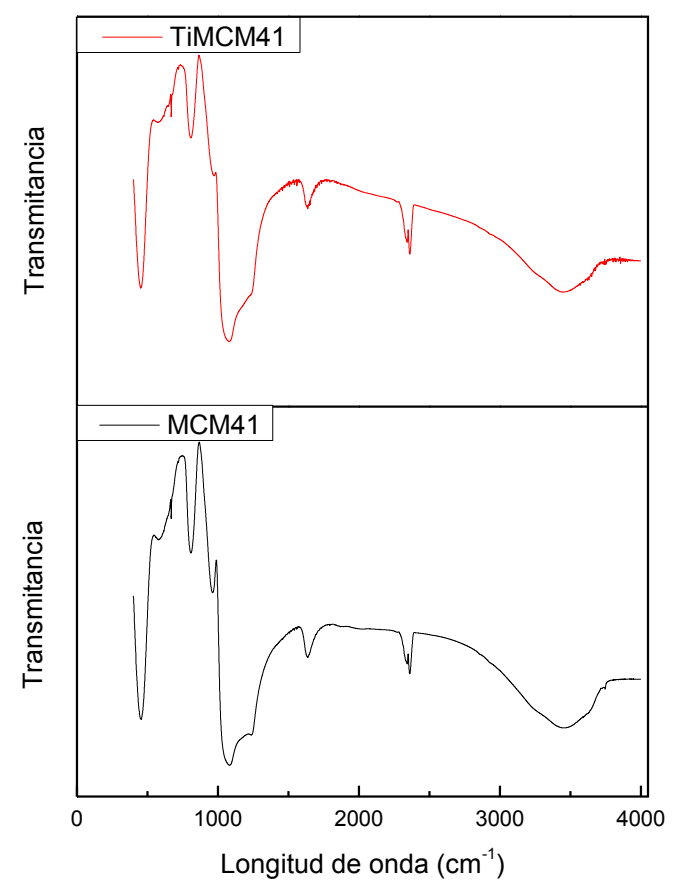

Figura 3: Espectros de FTIR

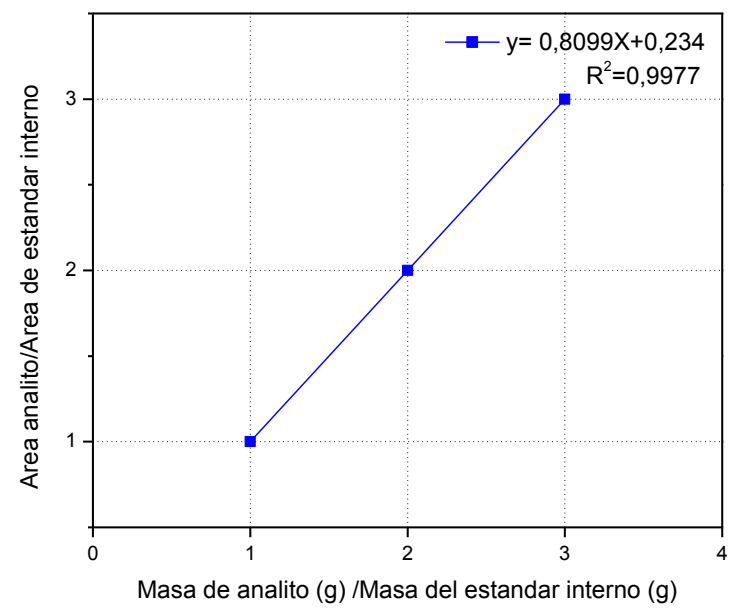

Figura 4: Curva de calibración. Factor de respuesta
En la Figura 2 se observa el UV-Vis RD del Ti-MCM-41. El material presenta una banda de absorción centrada a $243 \mathrm{~nm}$ correspondiente a las especies metálicas aisladas y tetraédricamente coordinadas con oxígeno (Liu et al., 2018). A $285 \mathrm{~nm}$ se encuentra una segunda banda asignada a especies de $\mathrm{Ti}$ con grados de coordinación superiores al tetraédrico, generadas por la hidratación de la estructura y/o la oligomerización incipiente de especies de Ti que forman nanoclusters Ti-O-Ti (Wang et al., 2012). El UV-Vis RD de la matriz silícea pura no se presenta ya que no existe absorción en la región analizada del espectro.

En la Figura 3 se presenta el FTIR de los materiales analizados. Tal como ha sido reportado (Zhao et al., 2020), se observa una banda de absorción a $458 \mathrm{~cm}^{-1}$ que se atribuye a la vibración de estiramiento del enlace Si-0. A $800 \mathrm{~cm}^{-1}$ y $1085 \mathrm{~cm}^{-1}$ se presentaron dos señales correspondientes a la vibración de estiramiento simétrico y asimétrico del Si-0 tetraédrico. La banda ubicada a $1240 \mathrm{~cm}^{-1} \mathrm{se}$ atribuye a los enlaces $\mathrm{Si}-\mathrm{O}-\mathrm{Si}$ y a $1640 \mathrm{~cm}^{-1}$ se observa una señal asociada a la presencia de $\mathrm{H}_{2} \mathrm{O}$. Las bandas ubicadas a $3460 \mathrm{~cm}^{-1} \mathrm{y}$ $970 \mathrm{~cm}^{-1}$ corresponden a los grupos Si-OH. Esta última señal puede estar asociada también a la vibración de los grupos Ti-O-Si generada por la incorporación del heteroátomo en el Ti-MCM-41, consistente con lo observado por UV-Vis RD. Por lo tanto, se deduce que en este material, la banda a $970 \mathrm{~cm}^{-1}$ corresponde a un solapamiento generado por los Si-OH y los heteroátomos (Cánepa et al., 2015).

Por último, el ICP indicó que la cantidad de Ti presente en el TiMCM-41 fue de 289.19 ppm que corresponde al 1.43\% p/p.

Identificación y cuantificación de reactivos y productos de reacción

El análisis obtenido por CG-MS permitió identificar los productos de reacción. Los mismos fueron 2,3-epoxihexanol y 2-hexenal. Para realizar la cuantificación de reactivos y productos, se aplicó el método de normalización de áreas empleando el factor de respuesta (Fr). Para obtener el valor del Fr del trans-2-hexen-1-ol, se realizó una curva de calibración utilizando pentanol como estándar interno. Para ello, se realizaron múltiples inyecciones de distintas relaciones volumétricas de trans-2-hexen-1-ol (analito)/estándar. Las áreas obtenidas de los análisis por triplicado se promediaron y luego se calculó la relación entre las áreas de analito y estándar. Por otro lado, se calculó la relación entre la masa del analito y el estándar para las muestras inyectadas. Estas relaciones fueron la ordenada y abscisa de la curva de calibración (Figura 4). La pendiente de la curva corresponde al factor de respuesta del analito para el detector FID, siendo de 0.81 para el alcohol analizado. Dado que no se cuenta con los productos de reacción como testigo y no pueden realizarse las curvas de calibración respectivas, el valor hallado de Fr para el alcohol fue utilizado para todos los productos de reacción debido a la similitud entre sus estructuras químicas. 
Evaluación catalítica

Se evaluaron los materiales MCM-41 y Ti-MCM-41 en la oxidación de trans-2-hexen-1-ol empleando una relación de sustrato/oxidante $=2$. A las 5 horas de reacción se obtuvieron conversiones de $1.69 \%$ mol para la matriz silícea pura mientras que con Ti-MCM-41 la conversión del sustrato fue de $4.26 \% \mathrm{~mol}$, lo que da cuenta que la incorporación de metales de transición tiene efecto en la actividad catalítica. A fines de mejorar los resultados obtenidos, se modificó la relación molar sustrato/oxidante empleando Ti-MCM-41. Como indica la Tabla 1, al utilizar una relación molar 4/1 la conversión de alcohol fue menor mientras que con una relación molar 1/1 se obtuvo la máxima conversión de trans-2hexen-1-ol, alcanzando un valor de 8.96\% mol. Así, se deduce que el incremento en la concentración del oxidante en el medio de reacción favorece la conversión del sustrato. Bajo estas condiciones, se logró un rendimiento máximo a 2hexenal y 2,3-epoxihexanol del 7.64\% mol y 1,32\% mol respectivamente.

\begin{tabular}{|c|c|c|c|c|c|}
\hline \multirow{2}{*}{$\begin{array}{c}\text { Relación molar } \\
\text { sustrato/oxidante }\end{array}$} & \multirow{2}{*}{$\begin{array}{c}\text { Conversión } \\
\text { (\% mol) }\end{array}$} & \multicolumn{2}{|c|}{ Selectividad (\% mol) } & \multicolumn{2}{|c|}{ Rendimiento (\% mol) } \\
\cline { 3 - 6 } & 2.85 & 8 Epóxido & Aldehído & Epóxido & Aldehído \\
\hline $4 / 1$ & 4.26 & 13.67 & 91.31 & 0.25 & 2.6 \\
\hline $2 / 1$ & 8.96 & 14.78 & 86.33 & 0.58 & 3.68 \\
\hline $1 / 1$ & 85.22 & 1.32 & 7.64 \\
\hline
\end{tabular}

Tabla 1: Efecto de la relación molar sustrato/oxidante en la oxidación de trans-2-hexen-1-ol a 5 h de reacción empleando Ti-MCM-

41.

\section{Conclusiones}

Se logró sintetizar y caracterizar materiales mesoporosos del tipo MCM-41. La actividad catalítica de estos sólidos se evaluó en la reacción de oxidación del trans-2-hexen-1-ol con $\mathrm{H}_{2} \mathrm{O}_{2}$. Se observó una mayor conversión de sustrato al emplear el material modificado con titanio. Con este último, y aplicando una relación molar de sustrato/oxidante $=1$, se alcanzó la mayor conversión, logrando un rendimiento aproximado a 2-hexenal del 9\% mol.

\section{Referencias}

Cánepa A., Chanquía C., Vaschetti V., Eimer G., y Casuscelli S. (2015). Biomass toward fine chemical products: Oxidation of a-pinene over sieves nanostructured modified with vanadium. Journal of Molecular Catalysis A: Chemical, 404-405, 65-73. http://dx.doi.org/10.1016/j.molcata.2015.04.009

Chanquía, C. M., Cánepa, A. L., Winkler, E. L., Rodríguez-Castellón, E. R., Casuscelli, S.G., Eimer, G. A. (2016). Nature of active vanadium nanospecies in MCM-41 type catalysts for olefins oxidation, Materials Chemistry and Physics, 175, 172179.

Du, X., y Rouseff, R. (2014). Aroma Active Volatiles in Four Southern Highbush Blueberry Cultivars Determined by Gas Chromatography-Olfactometry (GC-0) and Gas Chromatography-Mass Spectrometry (GC-MS), Journal of Agricultural and Food Chemistry, 62, 4537-4543. https://doi.org/10.1021/jf500315t

Farag, M. A., y W. Paré P. W. (2002). C6-Green leaf volatiles trigger local and systemic VOC emissions in tomato, Phytochemistry, 61, 545-554. https://doi.org/10.1016/S0031-9422(02)00240-6

Farzaneh, F., Zamanifar, E., Williams C. D. (2004). V-MCM-41 as selective catalyst for epoxidation of olefins and trans-2hexene-1-ol, Journal of Molecular Catalysis A: Chemical, 218, 203-209. https://doi.org/10.1016/j.molcata.2004.03.046

Gokbulut, I., y Karabulut, I. (2012). SPME-GC-MS detection of volatile compounds in apricot varieties, Food Chemistry, 132, 1098-1102. doi:10.1016/j.foodchem.2011.11.080 
Jia, W., Liu, T., Li, Q., y Yang, J. (2019) Highly efficient photocatalytic reduction of CO2 on surface-modified Ti-MCM-41 zeolite, Catalysis Today, 335, 221-227. https://doi.org/10.1016/j.cattod.2018.11.046

Liu, J., Fang, S., Jian, R., Wu, F., Jian, P. (2018). Silylated Pd/Ti-MCM-41 catalyst for the selective production of propylene oxide from the oxidation of propylene with cumene hydroperoxide, Powder Technology, 329, 19-24. https://doi.org/10.1016/j.powtec.2018.01.066

Ma, W., Zhao, L., y Xie Y. (2017). Inhibitory effect of (E)-2-hexenal as a potential natural fumigant on Aspergillus flavus in stored peanut seeds, Industrial Crops \& Products, 107, 206-210. http://dx.doi.org/10.1016/j.indcrop.2017.05.051

Neri F., Mari, M., Menniti A. M., Brigati, S., y Bertolini, P. (2006). Control of Penicillium expansum in pears and apples by trans-2-hexenal vapours, Postharvest Biology and Technology, 41, 101-108. doi:10.1016/j.postharvbio.2006.02.005

Pang, X. (2015). Biogenic volatile organic compound analyses by PTR-TOF-MS: Calibration, humidity effect and reduced electric field dependency, Journal of Enviromental Sciences, 32, 196-206. http://dx.doi.org/10.1016/j.jes.2015.01.013

Sienel G., Rieth R., Rowbottom K. T., (2008). Ullmann's Encyclopedia of Industrial Chemistry, Epoxides, John Wiley\&Sons.

Skoog, D. A., Holler, F. J., y Crouch, S. R. (2008). Principios de análisis instrumental. México, Cengage Learning.

Talens, P. (2003). Escriche, I., Martínez-Navarrete, N., y Chiralt, A. Influence of osmotic dehydration and freezing on the volatile profile of kiwi fruit, Food Research International, 36, 635-642. doi:10.1016/S0963-9969(03)00016-4

Wang S., Shi Y., y Ma X. (2012).Microwave synthesis, characterization and transesterification activities of Ti-MCM-41, Microporous and Mesoporous Materials, 156, 22-28. doi:10.1016/j.micromeso.2012.02.011

Wróblewska, A., Fajdek, A., Wajzberg, J., y Milchert E. (2009). Epoxidation of allyl alcohol over mesoporous Ti-MCM-41 catalyst, Journal of Hazardous Materials, 170, 405-410. doi:10.1016/j.jhazmat.2009.04.082

Yang G., Chen X., Wang X., Xing W., y Xu N. (2013). Nickel(II) complex anchored on MCM-41 for the epoxidation of styrene by oxygen, Chinese Journal of Catalysis, 34, 1326-1332. DOI: 10.1016/S1872-2067(12)60568-3

Zhao J., Zhang Y., Xu L., Tian F., Hu T., y Meng C. (2020).Weak base favoring the synthesis of highly ordered V-MCM-41 with well-dispersed vanadium and the catalytic performances on selective oxidation of benzyl alcohol. Chinese Journal of Chemical Engineering, 28, 1424-1435. https://doi.org/10.1016/j.cjche.2020.02.027 